Journal Club

Editor's Note: These short, critical reviews of recent papers in the Journal, written exclusively by graduate students or postdoctoral fellows, are intended to summarize the important findings of the paper and provide additional insight and commentary. For more information on the format and purpose of the Journal Club, please see http://www.jneurosci.org/misc/ifa_features.shtml.

\title{
How Working Memory Training Improves Emotion Regulation: Neural Efficiency, Effort, and Transfer Effects
}

\author{
Haakon Engen and Philipp Kanske \\ Department of Social Neuroscience, Max Planck Institute for Human Cognitive and Brain Sciences, 04103 Leipzig, Germany \\ Review of Schweizer et al.
}

Deficient regulation of emotion is a core problem across psychopathologies—such as depression, anxiety, and bipolar disorder (Gross, 2013) — that remains even after remission (Kanske et al., 2012). The development of better and more costeffective interventions aimed at improving emotion regulation abilities could offer substantial benefit to individuals and society at large. The main goal for such interventions is to achieve robust transfer effects, such that training on a specific task leads to more generalized improvement in emotion regulation. Achieving this is problematic because the relatively lowlevel processes that are most amenable to training, such as attention and working memory, are far less complex than the high-level processes that must be modified for the intervention to improve emotion regulation in real life. One of the great, but hitherto largely unfulfilled, promises of cognitive neuroscience is that it can help bridge such gaps by using brain data to identify the component neural systems of complex higher-level processes. As a consequence of extensive neuroimaging research on emotion, we are now in a position where such a decomposition can be undertaken, and mechanistic neural models of emotion regulation are pro-

Received May 18, 2013; revised June 18, 2013; accepted June 20, 2013.

Correspondence should be addressed to Philipp Kanske, Department of Social Neuroscience, Max Planck Institute for Human Cognitive and Brain Sciences, Stephanstr. 1a, 04103 Leipzig, Germany. E-mail: kanske@cbs.mpg.de.

DOI:10.1523/JNEUROSCI.2115-13.2013

Copyright $\odot 2013$ the authors $\quad 0270-6474 / 13 / 3312152-02 \$ 15.00 / 0$ posed (Ochsner et al., 2012). These models might lead to the development of interventions that improve emotion regulation abilities indirectly, by improving the function of its neural subsystems.

A recent example of this approach is the paper by Schweizer and colleagues (2013), showing that a short, inexpensive, and easily accessible regime of neurally targeted working memory (WM) training can lead to improvements in emotion regulation. The study is based on the observation that the neural networks supporting emotion regulation largely overlap with the frontoparietal "multiple-demand" network (Duncan, 2010) implicated in working memory performance. Accordingly, there may be a link between these two capacities, such that improvements in one will lead to improvements in the other by enhancing general functioning of the frontoparietal network. Schweizer et al. tested this hypothesis by having participants undergo a $20 \mathrm{~d}$ training program involving a daily 20-30 min performance of either a challenging dual n-back emotional WM task or a placebo training without an emotional WM component. Before and after training, participants underwent a scanning session during which they performed both the emotional WM task and a test of emotion regulation. In the latter, participants reduced their emotional reactions to film clips depicting emotionally aversive events using cognitive reappraisal. On the behavioral level, training related changes in emotional WM performance showed the hypothesized relationship with emotion regulation abili- ties: Participants who underwent the training performed better on the WM task and also reported that they were better able to reduce their negative affective responses. This relationship also held on the neural level, such that individuals who underwent the training showed greater activation change in the frontoparietal network when performing both the emotional WM task and the emotion regulation task. Thus, both on a neural and behavioral level, the results support the hypothesized transfer effect between emotional WM and emotion regulation, and demonstrate that this transfer is associated with changes in the functioning of the frontoparietal network. Interestingly, this relationship was such that the training led to decreased frontoparietal activation during the emotional WM task, but increases in frontoparietal activation during the emotion regulation task. Thus, the behavioral improvement in the two tasks seems to be supported by opposite changes in neural activation patterns. As discussed below, this observation might offer insights into the mechanism underlying transfer effects and into which component processes of emotion regulation were improved by the training.

What constitutes the neural signature of optimal processing is still a subject for debate (Neubauer and Fink, 2009). One major model is that of neural efficiency, which proposes that performance improvements should be associated with decreased activation, reflecting increased efficiency (i.e., less energy consumed in performing the task) of the underlying 
neural circuits. The opposing corticaleffort model, supported by much of the task-based neuroimaging literature, proposes that performance improvement is associated with increased activation, reflecting the increased ability to recruit the requisite neural circuits to perform a task. It has recently been proposed that these conflicting findings can be reconciled by considering the difficulty of the tasks being performed (Neubauer and Fink, 2009): A highly trained individual will be capable of performing a moderately difficult task with relative ease, leading to net deactivation relative to an untrained individual performing at the same level. A highly trained individual will also be more capable of sustained effort on a more difficult task, leading to a net increase of activation on such tasks. An example of this pattern is found in the Schweizer et al. (2013) study, where they show that, as participants' performance improved, enabling them to perform the emotional WM task at higher difficulty levels, activations were reduced during performance at the medium-difficulty level that constituted their performance ceiling before training. Additionally, activations associated with their new, higher-difficulty performance ceiling were similar to what had been observed at their pretraining mediumdifficulty performance ceiling. Thus, activation of the frontoparietal network was only apparent when participants were at their performance ceilings both before and after training.

Given the above considerations this suggests that the effect of the emotional WM task on emotion regulation stems from increased ability to engage the frontoparietal effort network to perform challenging tasks over time. In recent models of the neural architecture of emotion regulation via cognitive change, this network has been suggested to subserve both the monitoring of affective change and the maintenance of emotion regulation strategies over time (Ochsner et al., 2012). This is of note because success on the specific emotion regulation task used in the study depends on both the capacity to generate an appropriate cognitive change and to maintain this change over the course of a $30 \mathrm{~s}$ film clip. While hypothetical at present, these considerations suggest that what the training most likely achieved is improvement in participants' capacity to maintain regulation over time.

These results raise several questions for future research. First, it will be critical to test the maintenance hypothesis by investigating the temporal dynamics of the emotion regulation process itself. If the above reasoning is correct, one would expect the training effects to be more apparent in later stages of the regulation process, reflecting increased maintenance ability. Second, it would be interesting to see whether the effect of this training generalizes to other emotion regulation strategies, such as distraction, that do not rely so heavily on maintenance processes and have their effect at earlier stages of processing than cognitive change (Sheppes and Gross, 2011). It might be that emotional WM transfer effects are specific to modes of emotion regulation requiring deliberation and extended effort. Third, it is vital to gain a deeper understanding of how the beneficial effects of emotional WM on cognitive change are instantiated. Successful emotion regulation via cognitive change is a process of cortical-subcortical interactions (Wager et al., 2008). Based on the Schweizer et al. (2013) study, the subgenual ACC is playing an important role in mediating the effect of emotional WM training on subcortical structures. Connectivity-based methods could investigate this further by testing how the interaction of brain networks changes as a function of training. Finally, whether the training would be effective in clinical populations remains an open question. There is good reason to believe it would be, because affective disorders are often associated with working memory deficits, with concomitant abnor- malities in parts of the network targeted by Schweizer et al. (Townsend et al., 2010).

The Schweizer et al. (2013) study offers a first enticing example of how our knowledge of the brain has reached a stage at which we can begin to develop evidencebased interventions to improve mental health by considering the neural networks involved. In turn, this approach is greatly valuable for cognitive neuroscience, because it allows causal inferences about the role of network components for higher level functions.

\section{References}

Duncan J (2010) The multiple-demand (MD) system of the primate brain: mental programs for intelligent behaviour. Trends Cogn Sci 14: 172-179. CrossRef Medline

Gross JJ (2013) Emotion regulation: taking stock and moving forward. Emotion 13:359-365. CrossRef Medline

Kanske P, Heissler J, Schönfelder S, Wessa M (2012) Neural correlates of emotion regulation deficits in remitted depression: the influence of regulation strategy, habitual regulation use, and emotional valence. Neuroimage 61:686-693. CrossRef Medline

Neubauer AC, Fink A (2009) Intelligence and neural efficiency. Neurosci Biobehav Rev 33:1004-1023. CrossRef Medline

Ochsner KN, Silvers JA, Buhle JT (2012) Functional imaging studies of emotion regulation: a synthetic review and evolving model of the cognitive control of emotion. Ann N Y Acad Sci 1251:E1-E24. CrossRef Medline

Schweizer S, Grahn J, Hampshire A, Mobbs D, Dalgleish T (2013) Training the emotional brain: improving affective control through emotional working memory training. J Neurosci 33:5301-5311. CrossRef Medline

Sheppes G, Gross JJ (2011) Is timing everything? Temporal considerations in emotion regulation. Pers Soc Psychol Rev 15:319-331. CrossRef Medline

Townsend J, Bookheimer SY, Foland-Ross LC, Sugar CA, Altshuler LL (2010) fMRI abnormalities in dorsolateral prefrontal cortex during a working memory task in manic, euthymic and depressed bipolar subjects. Psychiatry Res 182:22-29. CrossRef Medline

Wager TD, Davidson ML, Hughes BL, Lindquist MA, Ochsner KN (2008) Prefrontal-subcortical pathways mediating successful emotion regulation. Neuron 59:1037-1050. CrossRef Medline 